\title{
Risco e resiliência familiar. A avaliação dos profissionais no contexto da
} proteção à criança

\section{Risk and family resilience. The evaluation of professionals in the context of child protection}

\author{
Cláudia Miranda, Ana Almeida \\ Universidade do Minho
}

\begin{abstract}
Resumo
A avaliação das famílias em risco psicossocial sob o prisma da resiliência familiar permite compreender as forças, recursos e estratégias que se constituem processos chave desenvolvidos pelas famílias na adversidade.

Através do Q-set da Resiliência familiar, ancorado no método $Q$-sort (Block, 2008) e no modelo da Resiliência Familiar (Walsh, 1998, 2005), 30 famílias de menores com medidas de promoção e proteção foram avaliadas pelos respetivos gestores de caso ( $\mathrm{n}=17$ profissionais). Os resultados confirmam a sobrevalorização do risco nestas famílias. Contudo, a ponderação da dinâmica risco-proteção favorece o reconhecimento de mecanismos de proteção imprescindíveis ao desenvolvimento de um funcionamento familiar resiliente.

Palavras chave: resiliência familiar, risco psicossocial, processos-chave, método Q-sort, risco-proteção
\end{abstract}

\begin{abstract}
The evaluation of families at psychosocial risk, under the perspective of family resilience, allows us to understand forces, resources and strategies that become key processes which are developed by families under adverse situations. Using the Q-set of the Family Resilience, anchored upon the Q-sort method (Block, 2008) and the model of family resilience (Walsh, 1998, 2005), 30 families with children referred to promotion and protection services, were evaluated by their respective case managers ( $\mathrm{n}=17$ professionals). The results confirm the overestimation of risk in these families. However, the consideration of the risk-protection dynamic elicits the recognition of which resources and protective mechanisms are essential strengths to the development of a resilient family functioning.

Keywords: family resilience, psychosocial risk, key processes, Q-sort method, risk-protection
\end{abstract}

Ao longo do seu ciclo vital, todas as famílias enfrentam situações difíceis e geradoras de instabilidade, decorrentes de acontecimentos normativos (e.g. saída de casa de um filho, morte de um familiar idoso...) e/ou não normativos (e.g. acidente, hospitalização, divórcio, desemprego...). O impacto destas situações negativas pode gerar desajustamentos significativos nas famílias que as vivenciam por comparação àquelas que não as experienciam. Felizmente, o efeito destes fatores de risco não é tão destrutivo quando se desencadeiam forças positivas ou protetoras - fatores de proteção ou de resiliência, que reduzem ou atenuam substancialmente o efeito adverso do risco (e.g. procura de apoio nas redes de suporte formal e informal; intensificação do controlo parental em ambientes de risco). De entre a diversidade familiar, as famílias em risco psicossocial, onde por definição várias circunstâncias aumentam o nível de adversidade, são também aquelas onde os processos de resiliência ganham a sua expressão máxima considerando que fazem emergir processos atípicos capazes de gerar desenvolvimento positivo na inadaptação (Luthar, 2006).

Risco e proteção são dimensões presentes que, numa perspetiva ecológica-sistémica e desenvolvimental, desenham as trajetórias de vida das famílias resilientes. E essencialmente, o conceito de resiliência familiar permite-nos olhar a família como um sistema dinâmico com capacidade para produzir mudanças positivas face à adversidade. Para Froma Walsh (2004), esta capacidade de responder a situações adversas resulta das interações no seio familiar e da influência que estas geram em cada indivíduo e na família como um todo único e singular. Segundo Walsh (2004), pensar os processos de resiliência remete para três domínios específicos do funcionamento familiar: (1) o sistema de crenças da família; (2) os padrões de organização; e (3) os processos de comunicação, essenciais para a coesão e o fortalecimento do sistema familiar, contribuindo decisivamente para a qualidade das interações familiares. Num jogo dinâmico entre domínios, o sistema de crenças é responsável por criar e atribuir significados às experiências da família. Os padrões organizacionais são constituídos pelas normas internas e externas que proporcionam uma estrutura e configuração únicas a cada família. Por último, os processos de comunicação congregam o meio através do qual aspirações, sentimentos e informações competem para facilitar a expressão, o compartilhar de ideias e de 
emoções, o apresentar soluções e o negociar estratégias de ação e de resolução de problemas.

A configuração da resiliência familiar como um constructo que conjuga duas dimensões - a adversidade de um nível considerável e a adaptação positiva (Luthar, 2006, p.742), acarreta dificuldades adicionais à avaliação da resiliência das famílias que, do ponto de vista metodológico, requer formatos e opções ajustados à especificidade do fenómeno, e que tendencialmente se têm traduzido em metodologias qualitativas. Segundo Cowan, Cowan \& Schultz (1996), as avaliações que tendem a centrar-se na apreciação do nível de risco das famílias resultam pouco compreensivas para avaliar e fazer emergir os níveis de adaptação das famílias, que requerem novas metodologias, mais completas e sofisticadas, capazes de contemplar os riscos e vulnerabilidades das famílias, como também as forças, os fatores de proteção e de resiliência que se combinam em vários domínios do sistema familiar, aumentando em diferentes aspetos o funcionamento adaptativo ou aliviando o inadaptativo. Não raras vezes, no trabalho com as famílias, os profissionais detêm frequentemente a perceção de que lidam com famílias difíceis, sendo que a avaliação tende sobretudo a centrar-se nos seus défices, vulnerabilidades ou fatores de risco, subestimando as suas competências e potencialidades.

Equacionando as exigências da avaliação da resiliência, o Q-set da Resiliência familiar nasce no âmbito de uma investigação intitulada "Resiliência Familiar e Risco Psicossocial - Estudo das perceções das famílias e dos profissionais que as acompanham" (Miranda, 2015), com o objetivo de compreender a resiliência familiar sob a perspetiva das famílias e dos profissionais. Baseado na metodologia Q-sort (Block, 2008) e no modelo de Resiliência Familiar de Walsh (1998, 2005), o Q-set da Resiliência Familiar surge como um instrumento de cariz nomotético e ideográfico para a avaliação da resiliência em famílias em risco psicossocial. Agrega fatores de risco e proteção e, graças à técnica do Q-sort, dá conta da interação destes fatores e do seu contributo no funcionamento adaptativo de cada família.

No presente artigo descrevemos a metodologia Q-sort, como uma ferramenta de avaliação quantitativa e qualitativa que se enquadra na categoria dos métodos mistos, procurando sublinhar a necessidade de aprofundar a compreensão das variáveis-chave na resiliência familiar (Black \& Lobo, 2008; Luthar \& Brown, 2007, citados por Walsh, 2012, p. 414). É também um objetivo deste estudo reforçar que a visão da intersubjetividade psicológica pode ser coadjuvada por indicadores explícitos, passíveis de tradução na linguagem das famílias e profissionais, não dispensando a comprovação científica e espelhando com rigor e transparência a multiplicidade de aspetos que caracterizam os contextos de risco das famílias.

\section{Método}

\section{Participantes}

Participaram no estudo 30 progenitoras de famílias heteroparentais ou monoparentais maternas, com filhos aos quais tinha sido aplicada uma medida de promoção e proteção "em meio natural de vida", designadamente de apoio junto dos pais ou de apoio junto de outro familiar com o qual residissem, com a finalidade de assegurar a permanência dos menores no seio da família de origem.

Da amostra fizeram também parte 17 profissionais de Comissões de Promoção de Crianças e Jovens (CPCJs) da região Norte do país que, enquanto gestores dos processos de promoção e proteção das famílias, eram detentores de informação ou conhecimento privilegiado acerca das mesmas.

\section{Instrumentos}

O Q-set da Resiliência familiar, desenvolvido no âmbito do estudo "Risco e Resiliência familiar: Perceções das famílias e dos profissionais que as acompanham" (Miranda, 2005), trata-se de um instrumento para a avaliação da resiliência familiar composto por 63 itens. A totalidade destes itens reflete no seu conjunto as dimensões e processos-chave da resiliência familiar descritos no modelo de Walsh (1998, 2005). Em conformidade com o método Q-sort, a técnica determina a distribuição dos itens num número fixo e pré-determinado de categorias incluídas num contínuo que, neste estudo, assume uma escala de Likert com sete pontos do mais caraterístico ao menos caraterístico da família em avaliação.

Tendo por base os modelos teóricos sobre a resiliência familiar, os itens descrevem quer fatores de risco (e.g. item 48 - Na nossa família falamos muito a criticar ou a culparmo-nos uns aos outros), quer fatores de proteção (e.g. item 4 - Acreditamos que falar sobre as situações difíceis com os filhos os prepara para a vida). Estes, encontram-se escritos em cartões pequenos de modo a facilitar o seu manuseamento e distribuição pelas categorias existentes, permitindo trocas e acertos entre categorias até se atingir a distribuição que condense melhor o perfil de cada família. Tomando como exemplo os dois itens que acima se referem, os mesmos podem refletir um fator de risco ou proteção dependendo da categoria/ponto da escala na qual venham a ser colocados. Se, por exemplo, o item 48 for colocado na categoria 2 (descreve muito mal a família) esta classificação pontua este fator de risco como ausente. Ao invés, se for colocado numa das categorias mais altas, este item vai refletir a presença do fator de risco na família.

\section{Procedimento}

O estudo decorreu junto das progenitoras, representantes das famílias com menores com medidas de promoção e proteção, e junto dos respetivos profissionais, gestores de caso das CPCJs, que concordaram participar. Após uma breve apresentação do estudo e do $Q$-set da resiliência familiar, procedeu-se à sua aplicação individual nos dois grupos. Quer a aplicação se realizasse junto de uma progenitora quer junto de um profissional, o procedimento geral consistia na distribuição do conjunto de itens do Q-set por sete categorias que variavam desde a mais descritiva ou "que caraterizava totalmente a família" (categoria 7) até à 
menos descritiva ou que "não caracterizava nada a família" (categoria 1). Considerando as orientações da metodologia Q-sort de Block (2008), os itens dispõem-se pelas 7 categorias segundo a curva normal gaussiana $(5,9,11,13,11,9,5 ; \mathrm{N}=63)$. A presente análise dos resultados centrou-se nas categorias extremas dos dois pólos da escala de sete pontos (categorias 7, 6, 1 e 2), considerando que os itens das categorias intermédias, de acordo com Block, são menos expressivos, não constituindo objeto de análise.

\section{Resultados}

A análise quantitativa e qualitativa dos Q-sorts das famílias e dos profissionais das CPCJs, permite compreender como se configura a resiliência nestas famílias, através de um contraste entre perspetivas.

Respeitando as orientações metodológicas de Block, e considerando que a resiliência familiar obriga a considerar a dupla proteção-risco, a análise reporta simultaneamente às duas extremidades da distribuição Q-Sort, configurando a resiliência a partir de duas condições: a) a proteção, que se carateriza pela presença de fatores de proteção e a ausência de fatores de risco; b) o risco que se carateriza pela presença de fatores de risco e a ausência de fatores de proteção.

$\mathrm{O}$ quadro 1 apresenta a frequência dos itens de risco e de proteção mais pontuados pelas famílias e pelos profissionais, nas quatro categorias dos dois pólos extremos, destacando aqueles que caracterizam a família "muito bem" e "totalmente" (categorias 6 e 7) e, inversamente, aqueles que caraterizam "muito mal" e "nada" (categorias 1 e 2). Para a proteção, evidenciam-se assim os itens de proteção mais pontuados nas categorias 6 e 7 como presentes ou característicos das famílias, e os itens de risco mais pontuados nas categorias 1 e 2 como ausentes ou não característicos (linha de corte $\mathrm{f} \geq 15$ ). Inversamente, o risco configura-se com base no conjunto de itens de risco mais pontuados nas categorias 6 e 7 como presentes ou característicos das famílias, e nos itens de proteção classificados nas categorias 1 e 2 como ausentes ou não descritivos (linha de corte $f \geq 8$ ).

$\mathrm{O}$ quadro 2 ilustra a apreciação qualitativa ou conceptual desses itens à luz das dimensões e processos chave do modelo de resiliência familiar de Walsh (1998, 2005).
Quadro 1.

Configurações da resiliência das famílias em risco psicossocial - análise quantitativa

\begin{tabular}{|c|c|c|c|c|}
\hline & \multicolumn{2}{|c|}{ Proteção } & \multicolumn{2}{|c|}{ Risco } \\
\hline & $\begin{array}{l}\text { Itens } \\
\mathrm{FP}^{1} \\
(\text { catg.6, } \\
7)^{\mathrm{f}^{*}}\end{array}$ & $\begin{array}{l}\text { Itens } \\
\mathrm{FR}^{2} \\
\text { (catg. } 1 \text {, } \\
2)^{\mathrm{f}^{*}}\end{array}$ & $\begin{array}{l}\text { Itens } \\
\mathrm{FR}^{2} \\
(\text { catg.6, } \\
7)^{\mathrm{f}^{* * *}}\end{array}$ & $\begin{array}{l}\text { Itens } \\
\mathrm{FP}^{1} \\
\text { (catg. 1, } \\
2)^{\mathrm{f}^{* *}}\end{array}$ \\
\hline Famílias & $\begin{array}{l}04^{15} \\
05^{18} \\
10^{17} \\
16^{20} \\
17^{15} \\
56^{15}\end{array}$ & $\begin{array}{l}31^{15} \\
38^{16} \\
51^{19} \\
57^{18}\end{array}$ & $\begin{array}{l}35^{10} \\
39^{8} \\
43^{9} \\
63^{9}\end{array}$ & $\begin{array}{c}12^{9} \\
24^{10}\end{array}$ \\
\hline $\begin{array}{l}\text { Profissi } \\
\text { onais }\end{array}$ & $\begin{array}{l}10^{16} \\
22^{21} \\
44^{15} \\
56^{15}\end{array}$ & $\begin{array}{l}07^{15} \\
33^{17} \\
34^{16} \\
38^{16} \\
48^{16} \\
51^{17}\end{array}$ & $\begin{array}{c}15^{8} \\
36^{9} \\
37^{10} \\
39^{12} \\
63^{8}\end{array}$ & $\begin{array}{c}42^{9} \\
52^{10} \\
57^{9} \\
58^{9} \\
61^{11}\end{array}$ \\
\hline
\end{tabular}

${ }^{1}$ FP- Fatores de Proteção; ${ }^{2}$ FR - Fatores de Risco

$*_{\mathrm{f} \geq 15} ; *_{\mathrm{f} \geq 8}$ 
Quadro 2.

Configurações da resiliência das famílias em risco psicossocial - análise qualitativa

\begin{tabular}{|c|c|c|}
\hline & Proteção & Risco \\
\hline Modelo & Dimensões/ & Dimensões/ \\
\hline Walsh & $\begin{array}{l}\text { Processos chave } \\
\text { (itens)* }\end{array}$ & $\begin{array}{l}\text { Processos chave } \\
\text { (itens)* }\end{array}$ \\
\hline Famílias & $\begin{array}{c}\text { Sistema Crenças: } \\
\text { Extrair significado } \\
\text { adversidade }\left(4^{\mathrm{a}} ; 5^{\mathrm{a}}\right) \\
\text { Perspetiva positiva } \\
\left(10^{\mathrm{a}}\right) \\
\text { Espiritualidade } \\
\left(16^{\mathrm{a}} ; 17^{\mathrm{a}}\right) \\
\text { Padrões } \\
\text { Organizacionais: } \\
\text { Coesão }\left(31^{\mathrm{b}} ; 38^{\mathrm{b}}\right) \\
\text { Processos } \\
\text { Comunicacionais: } \\
\text { Resolução } \\
\text { colaborativa } \\
\text { problemas } \\
\left(56^{\mathrm{a}} ; 57^{\mathrm{b}}\right) \\
\text { Expressão } \\
\text { emocional aberta } \\
\left(51^{\mathrm{b}}\right)\end{array}$ & $\begin{array}{l}\text { Sistema Crenças: } \\
\text { Perspetiva positiva } \\
\left(12^{\mathrm{c}}\right) \\
\text { Efeitos cumulativos } \\
\text { stress }\left(63^{\mathrm{d}}\right) \\
\text { Padrões } \\
\text { Organizacionais: } \\
\text { Flexibilidade }\left(24^{\mathrm{c}}\right) \\
\text { Coesão }\left(35^{\mathrm{d}} ; 39^{\mathrm{d}}\right) \\
\text { Recursos sociais e } \\
\text { económicos }\left(43^{\mathrm{d}}\right)\end{array}$ \\
\hline Profissionais & $\begin{array}{c}\text { Sistema Crenças: } \\
\text { Extrair significado } \\
\text { adversidade }\left(7^{\mathrm{b}}\right) \\
\text { Perspetiva positiva } \\
\left(10^{\mathrm{a}}\right) \\
\text { Padrões } \\
\text { Organizacionais: } \\
\text { Flexibilidade }\left(22^{\mathrm{a}}\right) \\
\text { Coesão }\left(33^{\mathrm{b}} ; 34^{\mathrm{b}} ;\right. \\
\left.38^{\mathrm{b}}\right) \\
\text { Recursos sociais e } \\
\text { económicos }\left(44^{\mathrm{a}}\right) \\
\text { Processos de } \\
\text { Comunicação: } \\
\text { Clareza }\left(48^{\mathrm{b}}\right) \\
\text { Expressão } \\
\text { emocional aberta } \\
\left(51^{\mathrm{b}}\right) \\
\text { Resolução } \\
\text { colaborativa } \\
\text { problemas }\left(56^{\mathrm{a}}\right)\end{array}$ & $\begin{array}{l}\text { Sistema Crenças: } \\
\text { Espiritualidade }\left(15^{\mathrm{d}}\right) \\
\text { Efeitos cumulativos } \\
\text { stress }\left(61^{\mathrm{c}} ; 63^{\mathrm{d}}\right) \\
\text { Padrões } \\
\text { Organizacionais: } \\
\text { Coesão }\left(36^{\mathrm{d}} ; 37^{\mathrm{d}} ; 39^{\mathrm{d}} ;\right. \\
\left.42^{\mathrm{c}}\right) \\
\text { Processos de } \\
\text { Comunicação: } \\
\text { Expressão emocional } \\
\text { aberta }\left(52^{\mathrm{c}}\right) \\
\text { Resolução colaborativa } \\
\text { problemas }\left(57^{\mathrm{c}} ; 58^{\mathrm{c}}\right)\end{array}$ \\
\hline
\end{tabular}

* Natureza dos itens: Fatores de proteção (FP) vs. Fatores de risco (FR);

${ }^{\mathrm{a}} \mathrm{FP}$ catg. 6,$7 ;{ }^{\mathrm{b}} \mathrm{FR}$ catg. 1,$2 ;{ }^{\mathrm{c}} \mathrm{FP}$ catg. 1,$2 ;{ }^{\mathrm{d}} \mathrm{FR}$ catg. 6,7

\section{Discussão}

Com base nos resultados obtidos e nas estatísticas descritivas realizadas, as descrições do funcionamento resiliente das famílias, na perspetiva das próprias e dos profissionais, comprovam que ambos os grupos reconhecem potencialidades nas famílias em risco psicossocial não as caracterizando somente com base em défices ou vulnerabilidades. $O$ conjunto de resultados reforça a ideia, já defendida na literatura, de que a resiliência familiar se configura com base na interação dinâmica entre fatores de risco e proteção. Conforme o peso que estas dimensões de risco e proteção assumem nas famílias, obtém-se uma configuração da resiliência familiar que se diferencia em dois padrões interativos. O padrão de proteção, associado à presença de fatores de proteção e à ausência de fatores de risco. E o padrão de risco, que inversamente contempla a presença de fatores de risco e a ausência de fatores de proteção.

Constata-se ainda que as perspetivas das famílias e dos profissionais se diferenciam quanto ao risco e proteção percecionados nas famílias em risco psicossocial. Para a configuração do padrão de proteção, as famílias identificam um maior número de fatores de proteção presentes ( $\mathrm{n}=6$ itens), enquanto os profissionais contrabalançam os resultados com uma maior identificação de fatores de risco ausentes $(n=6$ itens). $\mathrm{Na}$ configuração do padrão de risco, as classificações dos profissionais ganham destaque pela pontuação superior de fatores de risco como presentes e de fatores de proteção ausentes comparativamente ao número apontado pelas famílias. As descrições das famílias apontam para uma perceção mais atenuada dos fatores de risco, através das pontuações mais elevadas de características favoráveis ou adaptativas e menor expressão conferida aos défices ou às características associadas à inadaptação.

A forma como as famílias percecionam o risco poderá, por si só, ser uma determinante da resiliência (Pinheiro, 2004). A relativização da sua condição de risco a favor de forças e competências, sugere um funcionamento pautado por fatores pessoais ou crenças positivas que lhes permitirão encarar os problemas com uma maior confiança e segurança do que aquela que teriam se apenas percecionassem o risco nas suas vidas. Em contraste, a perspetiva dos profissionais reflete a tendência a darem uma maior expressão às características de risco, nem sempre contrabalançada pela valorização das características protetoras ou de resiliência nas famílias. Compreende-se que os profissionais detêm, pela sua formação e experiência, vetores orientadores mais racionais e padronizados que lhes conferirão uma maior capacidade de identificação e avaliação do risco, não significando, contudo, uma desconsideração das potencialidades ou uma sobrevalorização do risco que as famílias apresentam nas suas vidas. Formações académicas de cariz mais tradicionalistas associadas a expetativas sociais em torno do seu papel enquanto profissionais das CPCJs, cuja ação se espera célere e protetora das crianças em risco, precipitarão em avaliações e intervenções mais remediativas e imediatistas, focalizadas na identificação e minimização dos riscos, no lugar de abordagens positivas, com enfoque na prevenção e capacitação das famílias.

Sob o ponto de vista conceptual e tomando por base o modelo de Walsh, também as perspetivas das famílias e dos profissionais se diferenciam, para a maioria dos itens, quanto às dimensões e processos chave da resiliência familiar neles representados. As famílias valorizam sobretudo fatores protetores ao nível da dimensão "Sistemas de crenças". Contemplam crenças positivas assentes na construção compartilhada das experiências de crise com os filhos (item 4), na valorização da união familiar (item 5), no apoio e 
encorajamento por parte de outros (item 10), no encarar das crises como oportunidades de crescimento (item 17) e no cultivo da fé e espiritualidade (item 16). Outro processo chave muito representado pelas famílias alude à importância do envolvimento de todos "lá de casa" na resolução dos problemas (item 56), com valor protetivo na dimensão "Processos de comunicação". Dado que a proteção compreende ainda a identificação de fatores de risco percecionados como ausentes, as famílias reportam ausência de risco ao nível da gestão do conflito (item 57) e do uso indevido de humor na partilha emocional (item 51), da dimensão "Processos de comunicação". Não consideram a coesão familiar ameaçada por insatisfação nos papéis e práticas parentais (item 31), nem por alianças criadas com os filhos contra o outro cônjuge (item 38), o que se refletiria em défices ao nível da dimensão "Padrões organizacionais".

Na perspetiva dos profissionais, a configuração que a proteção assume, envolve um conjunto de elementos protetores que, embora em menor número, integram as três dimensões da resiliência familiar do modelo de Walsh. Na dimensão "Sistemas de crenças", e à semelhança das famílias, reconhecem a importância do apoio dos outros no encorajamento face à crise (item 10). Ao nível dos "Padrões organizacionais", destacam a manutenção das rotinas diárias para uma maior estabilidade familiar (item 22), assim como a capacidade de mobilizarem recursos junto da comunidade ou da família alargada (item 44). Tal como as famílias, reconhecem a capacidade de conversarem e de se apoiarem mutuamente na resolução dos problemas (item 56), da dimensão "Processos de comunicação". Em contrapartida, e dado a sua centralidade no risco destas famílias, identificam um maior número de fatores de risco ausentes, cuja ação se evidencia protetora ao nível das três dimensões do modelo da resiliência. $\mathrm{Na}$ dimensão "Padrões organizacionais" (itens 33, 34 e 38), evidenciam também a ausência de sentimentos de insatisfação ou culpabilização quanto aos papéis e práticas educativas parentais adotadas. $\mathrm{Na}$ dimensão "Sistema de crenças", a ausência da crença externalizante e negativa de associar o fracasso ou a adversidade a fatores externos (item7). Na dimensão "Processos de comunicação", não identificam constrangimentos quanto à clareza das mensagens transmitidas (item 48) nem quanto ao uso do humor na partilha de emoções (item 7).

$\mathrm{Na}$ análise da configuração do risco, observam-se igualmente discrepâncias entre grupos. Para as famílias, o risco situa-se na dimensão "Padrões organizacionais", apontando défices de estabilidade e na gestão flexível para lidar com as mudanças, através, por exemplo, da (re)definição de regras ou obrigações (item 24). Na dimensão "Sistemas de crenças", destacam uma crença negativa e externalizante que associa as situações de sucesso ou fracasso a fatores externos e não controláveis (item 12). Descrevem como ausentes fatores protetores que refletem a existência de desigualdades de poder na relação conjugal (item 39); o evitar do apoio da família alargada no cuidar dos filhos (item 35) e uma reduzida participação social (item 43), sendo evidente a dissonância destes dois últimos fatores com a perspetiva dos profissionais. Os profissionais identificam mais uma vez, défices ao nível das três dimensões do modelo de Walsh. Apontam, na dimensão "Processos de comunicação", problemas na comunicação e intimidade conjugais (item 52) e na resolução de problemas por não recurso a estratégias eficazes (item 58). Ao nível dos "Padrões organizacionais", descrevem pais pouco presentes na vida familiar e dos filhos, afetando a coesão familiar (item 42). No "Sistema de crenças", apontam a ausência de proteção ao nível dos efeitos cumulativos do stress, indicando famílias que percecionam as suas vidas como sofridas e trágicas (item 11) e que subvalorizam a fé ou a espiritualidade (item 15), detendo assim sistemas de crenças enfraquecidos no enfrentar das crises.

Os profissionais, pela experiência e formação que detêm, não só pontuam mais facilmente o risco, como avaliam as famílias de uma forma mais sistémica e multidimensional, numa análise reflexiva e ponderada ao nível das três dimensões do modelo de Walsh.

A análise conceptual dos resultados permite, assim, aceder a uma matriz dos fatores de risco e de proteção mais presentes e ausentes nestas famílias, podendo constituir-se um guia importante na avaliação e intervenção junto das famílias para a promoção de um funcionamento mais resiliente.

\section{Conclusão}

As famílias em risco psicossocial, com histórias de vida pautadas por crises e sentimentos de desânimo, impotência e frustração, evidenciam características positivas que as ajudam a enfrentar as adversidades. À ideia veiculada por Walsh $(1998,2005)$ de que nenhuma forma ou estilo familiar é inerentemente saudável ou disfuncional, é importante acrescentar que mesmo as famílias seriamente perturbadas podem mobilizar recursos internos ou externos para fortalecer a sua resiliência familiar, evidenciando grandes potencialidades quando desafiadas pela crise. As situações de crise constituem-se enquanto oportunidades para despertar recursos de coping ambientais e pessoais, que se revelam importantes moderadores e amortizadores dos efeitos negativos do risco (Gore e Eckenrode, 1994).

Assim, no lugar de avaliações e intervenções sociais unicamente focalizadas nos riscos e défices, a avaliação da resiliência familiar deverá constituir uma tarefa imprescindível dos serviços de apoio às famílias, pelas possibilidades que se colocam ao nível da moderação do impacto dos riscos e da reversibilidade das condições de adversidade com que as famílias se deparam, quando se identificam e se promovem também as suas forças e potencialidades. É com base nesta conceção que este estudo vem oferecer um instrumento que se poderá revelar útil no campo do estudo e da intervenção com as famílias. Assente numa metodologia ideográfica, centrada na pessoa, e oferecendo uma visão mais sistémica da resiliência, captando o binómio risco-proteção, o Q-set possibilita uma caracterização mais compreensiva da resiliência familiar, assim como 
das variáveis que contribuem para a sua determinação. Ao constituir um método nomotético, de classificação, que conjuga a análise das variáveis a um sistema de organização das mesmas, possibilita ainda a realização de contraste entre famílias, e entre estas e os profissionais que as acompanham ou outros elementos significativos das suas vidas, ajudando a desvelar as características que mais se associam às diferenças e o incremento de intervenções mais ajustadas.

Alcança-se, deste modo, um maior entendimento das configurações que a proteção e o risco assumem nas famílias, dando lugar a intervenções mais positivas, com base em forças, competências e no mobilizar de recursos ainda não utilizados pelas famílias. Além de uma parentalidade positiva, as intervenções familiares sistémicas e/ ou direcionadas especificamente ao casal, deverão contemplar também a promoção de processos de resiliência, entre os quais, a resolução colaborativa dos problemas, uma comunicação clara e direta, uma partilha emocional aberta e crenças mais positivas face ao futuro e ao enfrentar das crises.

\section{Referências}

Block, J. (2008). The Q-sort in carácter appraisal. Encoding subjective impressions of persons quantitatively. Washington: American Psychological Association. http://dx.doi.org/10.1037/11748-000

Cowan, P. A., Cowan, C. P. \& Schultz, M. S. (1996). Thinking about risk and resilience in families. In E. M. Hetherington \& E. A. Blechman (Eds.), Stress, coping and resiliency in children and families (1-38). New Jersey: Lawrence Erlbaum Associates.

Gore, S. \& Eckenrode, J. (1996). Context and process in research on risk and resilience. In R. J. Haggerty, L. R. Sherrod, N. Garmezy \& M. Rutter (Eds.), Stress, risk and resilience in children and adolescents: Processes, mechanisms and interventions (19-63). New York: Cambridge University Press.

Luthar, Suniya S. (2006). Resilience in development: A synthesis of research across five decades. In D. Cicchetti \& D. J. Cohen, (Eds), Developmental psychopathology, Vol. 3: Risk, disorder, and adaptation (2nd ed.) (739-795). Hoboken, NJ, US: John Wiley \& Sons.

Miranda, C. (2015). Resiliência Familiar e Risco Psicossocial: Estudo das perceções das Famílias e dos Profissionais que as acompanham. Mestrado em Estudos da Criança, Intervenção Psicossocial com Crianças, Jovens e Famílias, Instituto da Educação, Universidade do Minho, Braga, Portugal. http://hdl.handle.net/1822/40954

Pinheiro, D. P. N. (2004). A Resiliência em discussão. Psicologia em Estudo, 9(1), 67-75. http://dx.doi.org/10.1590/S1413-73722004000100009

Walsh, F. (1998). Strengthening Family Resilience. New York, London: The Guilford Press.

Walsh, F. (2004). Resiliencia Familiar: Estrategias para su fortalecimiento. Buenos Aires: Amorrortu Editores.

Walsh, F. (2005). Fortalecendo a Resiliência Familiar. São Paulo: Roca.
Walsh, F. (2012). Family resilience: Strengths forged through adversity. In: Walsh, F. (Org.). Normal Family Processes. 4th ed. New York: Guilford Press, p. 339-427. 